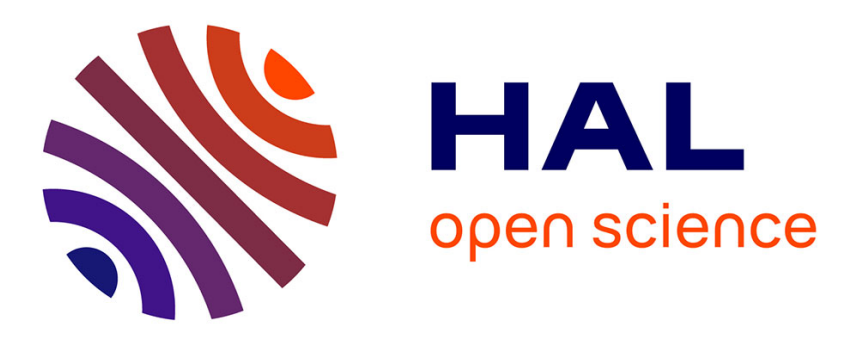

\title{
Image-based adaptive optics for two-photon microscopy
}

Delphine Débarre, Edward J. Botcherby, Tomoko Watanabe, Shankar

Srinivas, Martin J. Booth, Tony Wilson

\section{To cite this version:}

Delphine Débarre, Edward J. Botcherby, Tomoko Watanabe, Shankar Srinivas, Martin J. Booth, et al.. Image-based adaptive optics for two-photon microscopy. Optics Letters, 2009, 34, pp.2495. 10.1364/OL.34.002495 . hal-00681924

\section{HAL Id: hal-00681924 \\ https://hal.science/hal-00681924}

Submitted on 28 Oct 2013

HAL is a multi-disciplinary open access archive for the deposit and dissemination of scientific research documents, whether they are published or not. The documents may come from teaching and research institutions in France or abroad, or from public or private research centers.
L'archive ouverte pluridisciplinaire HAL, est destinée au dépôt et à la diffusion de documents scientifiques de niveau recherche, publiés ou non, émanant des établissements d'enseignement et de recherche français ou étrangers, des laboratoires publics ou privés. 


\title{
Image-based adaptive optics for two-photon microscopy
}

\author{
Delphine Débarre, ${ }^{1,2, *}$ Edward J. Botcherby, ${ }^{1}$ Tomoko Watanabe, ${ }^{3}$ Shankar Srinivas, ${ }^{3}$ \\ Martin J. Booth, ${ }^{1}$ and Tony Wilson ${ }^{1}$ \\ ${ }^{1}$ Department of Engineering Science, University of Oxford, Oxford OX1 3PJ, United Kingdom \\ ${ }^{2}$ Current address: Laboratory for Optics and Biosciences, CNRS UMR 7546 INSERM U696, Ecole Polytechnique, \\ 91128 Palaiseau, France \\ ${ }^{3}$ Department of Physiology, Anatomy and Genetics, University of Oxford, Oxford OX1 3QX, United Kingdom \\ *Corresponding author: delphine.debarre@polytechnique.edu
}

Received April 23, 2009; revised June 15, 2009; accepted June 21, 2009;

posted July 17, 2009 (Doc. ID 110522); published August 13, 2009

We demonstrate wavefront sensorless aberration correction in a two-photon excited fluorescence microscope. Using analysis of the image-formation process, we have developed an optimized correction scheme permitting image-quality improvement with minimal additional exposure of the sample. We show that, as a result, our correction process induces little photobleaching and significantly improves the quality of images of biological samples. In particular, increased visibility of small structures is demonstrated. Finally, we illustrate the use of this technique on various fresh and fixed biological tissues. () 2009 Optical Society of America OCIS codes: $110.1080,010.7350,180.2520,180.6900,180.4315,170.3880$.

Two-photon excited fluorescence (2PEF) [1] is a widely used technique to obtain 3D images of thick biological specimens. One of its main advantages is that the image quality is only weakly affected by scattering up to a depth of several scattering lengths. Nevertheless, images still degrade quickly with depth, as tissues often induce excitation wavefront aberrations that detrimentally affect the image intensity and resolution, hence the use of an adaptive element, such as a deformable mirror (DM), to correct for these aberrations.

Although a wavefront sensor can be used for measuring aberrations at the focal spot [2], indirect measurement also provides a robust way of assessing them [3]. In sensorless schemes, the correction is optimized by applying a set of trial aberrations and measuring an image-quality metric $M$ for each of them until sufficient information is obtained on the initial aberration. The resulting additional sample exposure critically depends on the efficiency with which information is gained through the trial aberration sequence; practical implementation thus requires a model-based, optimized choice of $M$ and of the modes describing the aberrations. This approach has already proven successful in confocal [4] and more recently structured illumination (SI) microscopy [5].

Here we demonstrate that the approach can be extended to $2 \mathrm{PEF}$ microscopy. To determine appropriate parameters for correction, we used an approach recently developed for SI microscopy. First we selected a suitable metric $M$ that reaches a maximum in the absence of aberration, namely the mean image intensity. We subsequently investigated the dependence of $M$ on the pupil plane aberrations. Assuming that the sample spatial frequency content does not vary strongly over the thickness of the focal volume, it can be calculated that

$$
M \approx M_{0}-\sum_{i, j} a_{i} a_{j}\left\langle X_{i}, X_{j}\right\rangle,
$$

where $\left\{X_{i}\right\}$ is a set of modes describing the aberrations in the objective pupil plane, $a_{i}$ is the aberration amplitude in mode $i$, and

$$
\begin{aligned}
\left\langle X_{i}, X_{j}\right\rangle \propto & \int_{-\infty}^{+\infty} \int_{|\mathbf{m}| \leqslant 2}\left(\int_{C(\mathbf{m})} e^{-j \Delta \Phi_{d}} \int_{C(\mathbf{m})} \Delta X_{i} \Delta X_{j} e^{j \Delta \Phi_{d}}\right. \\
& \left.-\int_{C(\mathbf{m})} \Delta X_{i} e^{-j \Delta \Phi_{d}} \int_{C(\mathbf{m})} \Delta X_{j} e^{j \Delta \Phi_{d}}\right) \mathrm{d} \mathbf{m} \mathrm{d} z,
\end{aligned}
$$

where $C(\mathbf{m})$ is defined by $|\mathbf{r}-\mathbf{m}| \leqslant 1$ and $|\mathbf{r}| \leqslant 1, \Delta X_{i}$ $=X_{i}(\mathbf{r}-\mathbf{m})-X_{i}(\mathbf{r}), \mathbf{r}$ is the pupil plane coordinate, $\mathbf{m}$ is the normalized spatial frequency, and $\Delta \Phi_{d} \propto z(\mid \mathbf{r}$ $-\left.\mathbf{m}\right|^{2}-|\mathbf{r}|^{2}$ ) describes defocus [6]. As shown in [5], avoiding crosstalk between the corrections of each mode requires using an appropriate rotation of $\left\{X_{i}\right\}$ to define a new set $\left\{Y_{i}\right\}$ such that

$$
\left\langle Y_{i}, Y_{j}\right\rangle=\beta_{i} \delta_{i j},
$$

where $\beta_{i}$ is the eigenvalue for mode $Y_{i}$ and $\delta_{i j}$ is the Kronecker delta. We could thus use a quadratic maximization algorithm using $2 N+1$ measurements to correct for $N$ aberration modes and, contrary to previous schemes using random actuator combinations [3], to ensure that no tip, tilt, or defocus was induced during correction. The experimentally determined $\left\{Y_{i}\right\}$ are plotted in Fig. 1(b) for a initial set $\left\{X_{i}\right\}$ of 11 Zernike modes $(z=5$ to 15 , indexing scheme detailed in [7]).

We implemented our correction scheme on a homebuilt 2PEF microscope incorporating a DM (BostonMicromachinesCorp., Multi-DM, 3.5- $\mu \mathrm{m}$ stroke), galvanometer mirrors (GSI Lumonics), a photoncounting photomultiplier tube (SensTech), and a 60 $\times, 1.2$ NA water immersion objective (Olympus) [Fig. 
(a)

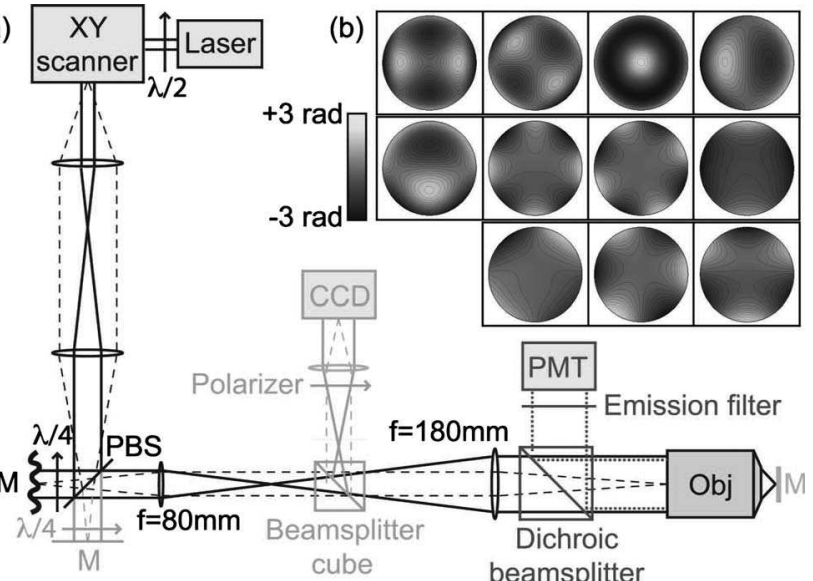

Fig. 1. Experimental setup: $\lambda / 2(\lambda / 4)$, half (quarter) wave plates; PBS, polarizing beam splitter; $M$, flat mirrors. Black solid lines, excitation path; dotted lines, emission path; gray lines, DM characterization path. Dashed lines show the conjugated Fourier planes. (b) Experimentally determined aberration modes $Y_{i}$ used for correction.

1(a)]. Excitation light overfilling the objective back aperture was provided by a pulsed Ti:sapphire laser (Spectra Physics) centered at $850 \mathrm{~nm}$ in all the experiments. Mirror control, data acquisition, and realtime image analysis were performed with LabView. Mirror characterization was performed as described elsewhere [8].

We first performed aberration correction on fresh mouse intestine tissue expressing a cyan fluorescent protein (CFP). Results for 11 corrected modes [shown in Fig. 1(b)] are displayed in Fig. 2. Although imaging was performed at shallow depths $(0$ to $80 \mu \mathrm{m})$, correction increases the $2 \mathrm{PEF}$ signal by about $50 \%$. To test the efficiency of our algorithm, we performed successive rounds of correction on the same sample, each using $2 N+1$ measurements. As expected, the quality of images did not improve for the second and subsequent rounds, showing that optimum correction was reached after a single iteration.

We then analyzed the influence of this correction on the properties of images. Although the $2 \mathrm{PEF}$ signal increases globally, a change in the contrast of small features is also observed owing to better transmission of high spatial frequencies; for small total aberration amplitude $a$, the peak intensity of pointlike features varies as the squared Strehl ratio, hence as $1-2 a^{2}$ [6], whereas the signal from a bulk sample varies as $\sim 1-0.77 a^{2}$ [as calculated from the eigenvalues $\beta_{i}$; see Eq. (3)]. As an example, we imaged a fresh mouse liver sample expressing an ubiquitous CFP providing a homogenous background with additional endogenous fluorescence observed in pointlike objects. The relative increase of these two signals after correction is plotted in Fig. 3(a), along with the total corrected aberration as a function of depth; contrary to the bulk fluorescence signal, the contrast of pointlike objects is significantly increased (over $100 \%$ increase for 0.9 rad total aberration).

It should also be noted that we can adapt the imaging parameters so as to limit sample illumination and the time required for correction. Indeed, correc-

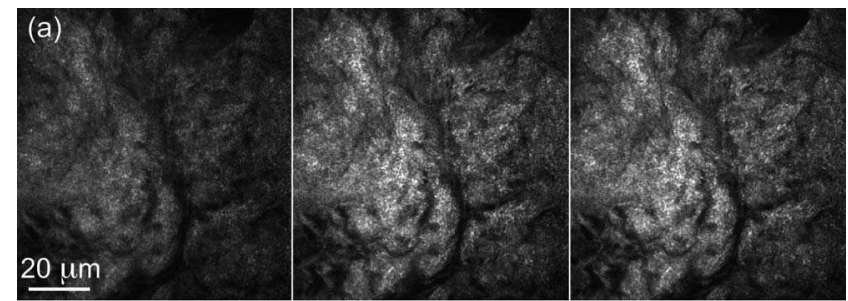

(b)

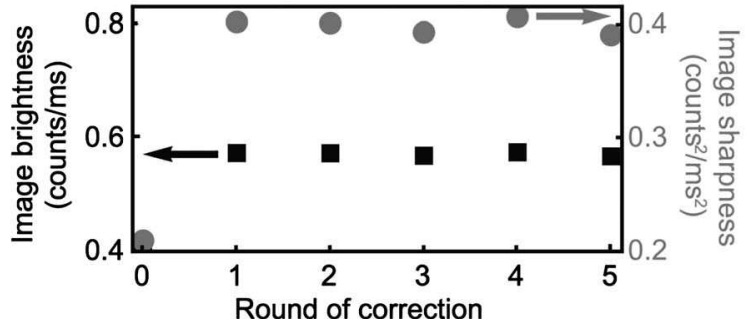

Fig. 2. (a) Fresh mouse tissue before (left) and after one (middle) and five (right) iterations of the correction algorithm. (b) Image intensity and sharpness of the same sample as a function of the number of iterations.

tion can be performed with a lower number of detected photons, and we accordingly decreased both the pixel dwell time (here by a factor $\approx 2.5$ ) and the number of pixels (by a factor $\approx 3$ ), so that the imaging time was 20-fold reduced [see inset in Fig. 3(b)]. Since 23 images were required here to correct for the aberrations, the total sample illumination was only about doubled, and as the correction algorithm itself is much quicker on a standard computer than the image acquisition, the time required for correction is essentially of the order of the acquisition time of a good-quality image. Here acquiring a corrected image thus required the same time and total exposure as an uncorrected image with a doubled acquisition time [Fig. 3(b), respectively middle and bottom], which for the deepest images provided the same intensity from pointlike objects but a much higher bulk signal, hence a lower visibility for small objects. This demonstrates the benefit of correcting for aberrations instead of increasing the excitation intensity or the pixel dwell time.

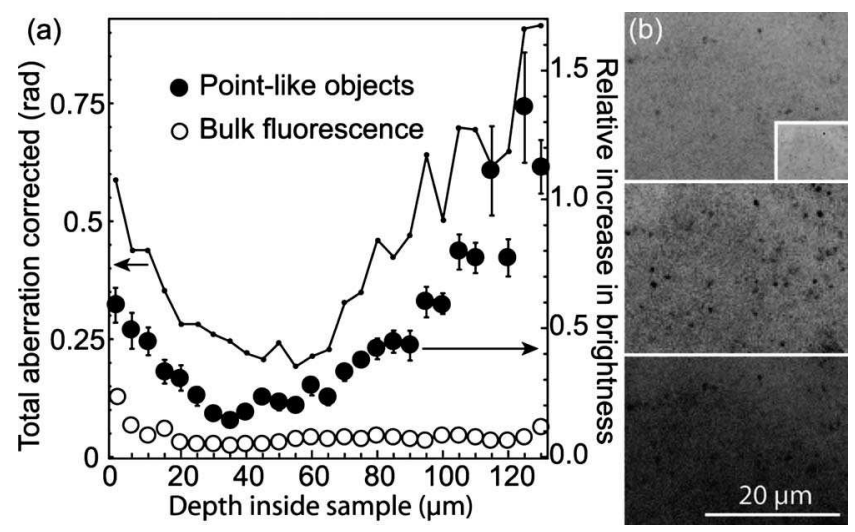

Fig. 3. (a) Visibility improvement of small structures after correction. (b) Corresponding image of a fresh mouse liver sample $100 \mu \mathrm{m}$ below the tissue surface before correction (top), after correction (middle), and before correction with an acquisition time twice longer (bottom). Inset, image of the same sample used for correction (same scale and color scale). Inverted contrast. 


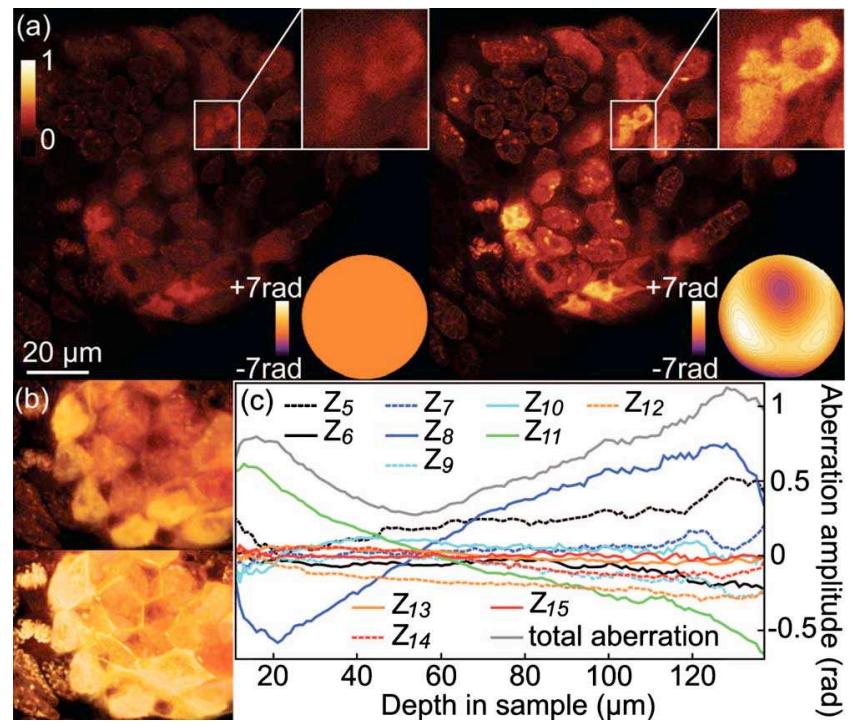

Fig. 4. (Color online) Depth-resolved correction on a mouse embryo. The two images (left, before; right, after correction) share the same color scale. Image size is $\approx 720$ $\times 640$ pixels. Insets, correction phase applied. (b) (Media 1, low res; Media 2, high res) 3D reconstruction of a $30 \times 40$ $\times 40 \mu \mathrm{m}^{3}$ region of the embryo. (c) Aberration amplitude in Zernike modes as a function of depth. The objective correction collar was set so as to optimize image quality at a depth of $\approx 50 \mu \mathrm{m}$ in the sample.

Finally, by measuring the depth intensity profile of the corrected images we could estimate the scattering mean-free path in the sample as $\approx 90 \mu \mathrm{m}$ at $850 \mathrm{~nm}$ [9]. This shows that even at depth of 1.5 scattering lengths, our method provides a robust aberration estimate that permits improvement of the image quality.

We demonstrated the use of our setup for 3D imaging on a fixed mouse embryo expressing green fluorescent protein (GFP) in a group of epithelial cells (Hex-GFP transgene) and with 4',6-diamidino-2phenylindole (DAPI)-stained DNA. At our excitation wavelength, both dyes could be excited, although the DAPI signal was much weaker. Images obtained for a plane containing GFP-stained cells and a 3D reconstruction of the deepest $40 \mu \mathrm{m}$ of the embryo (Media 1, low res; Media 2, high res) are displayed in Fig. 4; each shows improved intensity and resolution after correction. As shown in Fig. 4(c), aberration is mostly significant in three Zernike modes [astigmatism $\left(Z_{5}\right.$, $\left.Z_{6}\right)$, coma $\left(Z_{7}, Z_{8}\right)$ and first-order spherical $\left.\left(Z_{11}\right)\right]$. These values were obtained by converting the calculated correction from 2PEF modes [Fig. 1(b)] into Zernike modes. Aberration amplitude variations are strongly affected by the sample structure (see, for example, the change in the coma curve when penetrating the embryo epithelium, at depths $\approx 20 \mu \mathrm{m}$ and $\approx 130 \mu \mathrm{m}$ ), showing that at least part of the measured aberrations originates from the sample.

Figure 4 also shows a strong variation with depth of aberration, thus demonstrating the benefit of our method, which provides an axial resolution equal to the one of the imaging process itself. Similarly though, it should be noted that throughout this Letter, we calculated the optimal correction over the entire acquired image, thus mitigating the local $x y$ variations of aberration. As a result, as soon as spatial variations of aberration within the image existed, the correction was not optimized on every point of the image but rather was a mean over the image of local aberrations weighted by the local signal intensity in the absence of aberration. In most tissues, we found by performing correction on several subimages that this lateral variation was negligible, but in certain cases (such as the mouse embryo, Fig. 4), further accuracy would be gained by laterally adjusting the correction. This, however, could be done only at the expense of an increase in sample exposure, and benefit of this more accurate correction should be evaluated for the imaged sample. Similarly, depth sampling for aberration measurement should be adjusted to the scale of the aberration variations, as correction update might not be necessary for each imaging plane.

In conclusion, we have introduced a new imagebased technique to correct for aberrations in a $2 \mathrm{PEF}$ microscope and demonstrated its use for visualizing typical biological dyes within live samples. We have shown how this technique can provide a fast, reliable estimate of the aberration in scattering samples with a reduced number of measurements and thus limited additional exposure. Together, these points indicate that our scheme is well suited for biological imaging, where photobleaching, phototoxicity, time resolution, and scattering are limiting factors for most applications. In the future, this technique should prove helpful for imaging thick biological samples, e.g., in the fields of neurobiology or embryology.

D. Débarre was a Human Frontier Science Program Long-Term Fellow. M. J. Booth was an Engineering and Physical Sciences Research Council (EPSRC) Advanced Research Fellow (EP/E055818/1). This work was supported by a grant from the Biotechnology and Biological Sciences Research Council (BBSRC) (BBE0049461).

\section{References}

1. W. Denk, J. H. Strickler, and W. W. Webb, Science 248, 73 (1990).

2. M. Rueckel, J. Mack-Bucher, and W. Denk, Proc. Natl. Acad. Sci. USA 103, 17137 (2006).

3. P. N. Marsh, D. Burns, and J. M. Girkin, Opt. Express 11, 1123 (2003).

4. M. J. Booth, M. A. A. Neil, R. Juškaitis, and T. Wilson, Proc. Natl. Acad. Sci. USA 99, 5788 (2002).

5. D. Debarre, E. J. Botcherby, M. J. Booth, and T. Wilson, Opt. Express 16, 9290 (2008).

6. T. Wilson and C. J. R. Sheppard, Theory and Practice of Scanning Optical Microscopy (Academic, 1984).

7. M. A. A. Neil, M. J. Booth, and T. Wilson, J. Opt. Soc. Am. A 17, 1098 (2000).

8. M. J. Booth, T. Wilson, H.-B. Sun, T. Ota, and S. Kawata, Appl. Opt. 44, 5131 (2005).

9. P. Parsa, S. L. Jacques, and N. S. Nishioka, Appl. Opt. 28, 2325 (1989). 